\title{
A CONSTRUCTIVE METHOD FOR NUMERICALLY COMPUTING CONFORMAL MAPPINGS FOR GEARLIKE DOMAINS
}

\section{INTRODUCTION}

The Riemann mapping theorem asserts that the open unit disk $\mathcal{D}=$

$\{z|| z \mid<1\}$ is conformally equivalent to each simply connected domain $\mathcal{G}$ in the complex plane, whose boundary consists of at least two points, i.e., there exists a function $f$, analytic and univalent function on $\mathcal{D}$, such that $f$ maps $\mathcal{D}$ onto $\mathcal{G}$. More precisely, if $d_{o}$ is an arbitrary point in $\mathcal{D}$ and $g_{o}$ is an arbitrary point in $\mathcal{G}$, then the Riemann mapping theorem asserts that there exists a unique conformal mapping $f$ of $\mathcal{D}$ onto $\mathcal{G}$ such that $f\left(d_{o}\right)=$ $g_{o}$ and $f^{\prime}\left(d_{o}\right)>0$. If the boundary of $\mathcal{G}$ is piece-wise analytic and $g_{1}$ is a point on the boundary of $\mathcal{G}$, then the uniqueness assertion of the Riemann mapping theorem can be reformulated alternately as the statement that there exists a unique conformal mapping $f$ of $\mathcal{D}$ onto $\mathcal{G}$ such that $f\left(d_{o}\right)=g_{o}$ and $f(1)=g_{1}$.

The problem of constructing the explicit conformal mapping guaranteed by the Riemann mapping theorem is usually difficult, even numerically. There are constructive proofs [He] of the Riemann mapping theorem, but, because of their general nature, they often converge only slowly to the desired solution.

For polygonal domains, i.e., simply connected domains bounded by segments of straight lines, there is a well-known representation formula, called the Schwarz-Christoffel formula (see [Ne], p. 189), which the conformal mapping functions must satisfy. The difficulty with using the Schwarz-Christoffel formula to construct the conformal mapping for an explicit polygonal domain has always been that the formula contains unknown parameters, called the accessory parameters, which have to be determined before the mapping func- 
tion can be computed. D. Gaier in his monograph [Ga] on conformal mapping surveyed several methods which had been proposed for solving the accessory parameter problem and proposed a method for reducing the problem to a system of nonlinear equations. In 1980 L. N. Trefethen [Tr] devised an effective procedure for solving the accessory parameter problem and, hence, constructing the conformal mapping function for a polygonal domain. Trefethen used the geometry of the polygonal domain to construct a constrained system of nonlinear equations, the solution set of which would be the accessory parameter set.

In [Ba \& Pe], following work of A. W. Goodman [Go], necessary and sufficient conditions were given for representing the conformal mapping function which would map $\mathcal{D}$ onto a image domain $\mathcal{G}$ which is gearlike. A gearlike domain is a simply connected domain which contains the origin and is bounded by arcs of circles centered at the origin and by segments of straight lines through the origin. (See Figure 1.) We note that the logarithmic image of a gearlike domain is a periodic polygonal domain. Hence, one possible method for solving the conformal mapping problem for a gearlike domain would be to apply an adaption of Trefethen's method to the logarithmic image. In this paper we propose to directly use the representation formula for gearlike functions and a development motivated by the work of Trefethen to construct a procedure for numerically computing the conformal mapping function for a gearlike domain. The kernel of that procedure is an algorithm which prescribes a system of nonlinear equations whose solution set is the unknown accessory parameter set for the gearlike domain being considered. 


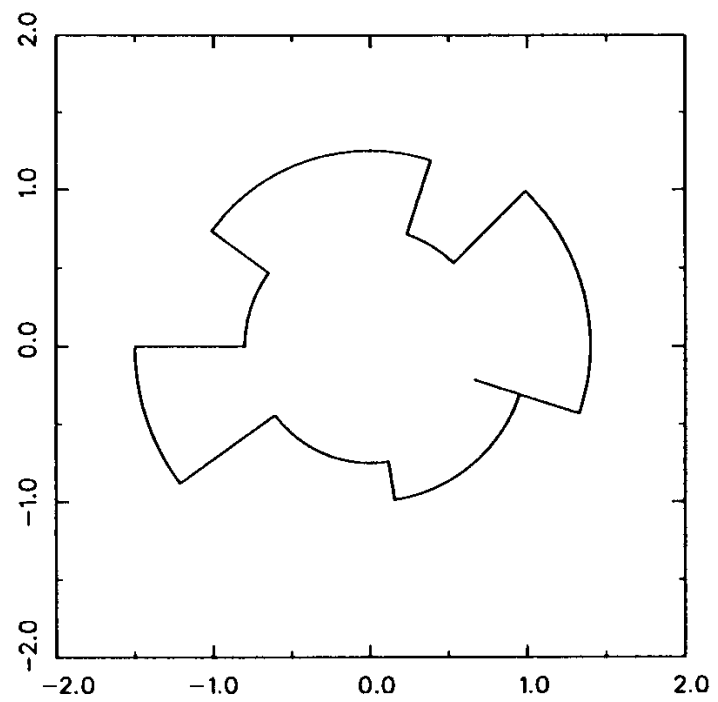

Figure 1

We will give several examples using this procedure for constructing both the forward mappings from $\mathcal{D}$ to explicit gearlike domains $\mathcal{G}_{i}$ and the inverse mappings from the domains $\mathcal{G}_{i}$ back to $\mathcal{D}$. We will conclude with an application which shows that a coefficient conjecture of R. W. Barnard [Ba] fails.

There are several recent references which give alternate constructions for related conformal mapping problems. K. P. Jackson and J. C. Mason [Ja \& Ma] treated a problem of crack stress around holes in two-dimensional plates by mapping the crack region conformally to the exterior to the unit circle. They used an adaption, made by Trefethen [Tr, 83], of the SCPACK programs Trefethen developed for solving Schwarz-Christoffel mapping problems. Recently, P. Bjørstad and E. Grosse [Bj \& Gr] devised a method for constructing the conformal mapping functions (from the unit disk) to circular arc polygons. A circular arc polygon is a polygon where the sides of the polygon are allowed to be general arcs of circles. Their method applies an o.d.e. solver to a specific second order differential equation which represents 
the circular arc polygon mapping function.

\section{GEARLIKE DOMAINS}

Let $\mathcal{G}$ be a gearlike domain in the complex plane with $n$ sides. Let $w_{k}, 1 \leq$ $k \leq n$, denote the vertices of $\mathcal{G}$, some of which may lie at infinity. For convenience, we will also denote $w_{n}$ as $w_{0}$. For each finite vertex $w_{k}$ let $\pi \alpha_{k}$ denote the interior angle at $w_{k}$ and let $\pi \beta_{k}$ denote the exterior angle at $w_{k}$. By definition $\alpha_{k}$ and $\beta_{k}$ satisfy the relation $\alpha_{k}+\beta_{k}=1$. For each infinite vertex $w_{k}$ set $\beta_{k}=1$. We note, in this latter case, that $\pi \beta_{k}$ is generally not the exterior angle at $w_{k}$.

For example, in Figure 2 at the corner vertices $w_{3}$ and $w_{6}$ we have $\beta_{3}=\frac{1}{2}$ and $\beta_{6}=-\frac{1}{2}$. At $w_{2}$, the tip of a slit, the external angle is $-\pi$, so $\beta_{2}=-1$.

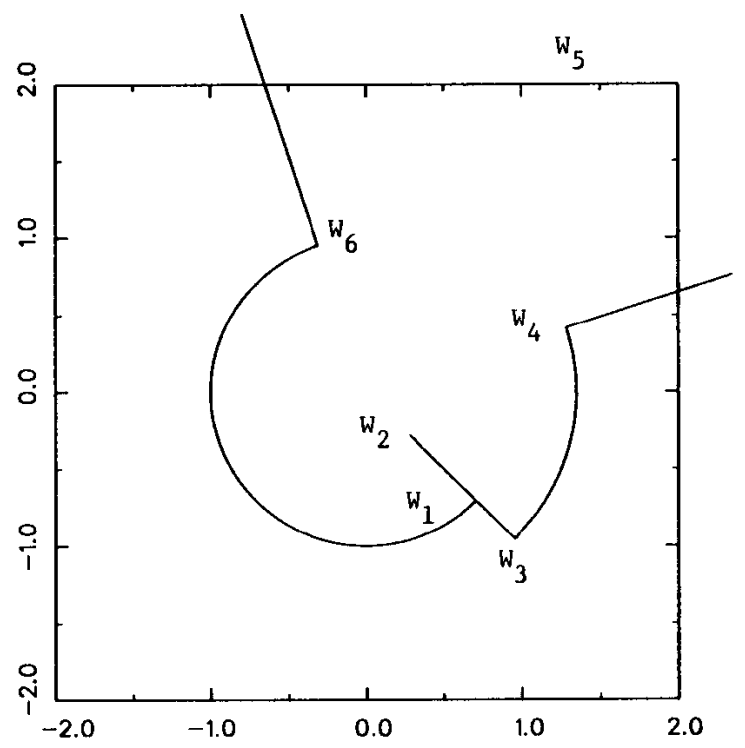

Figure 2

In $[\mathrm{Ba} \& \mathrm{Pe}]$ we showed that the following representation formula de- 
scribes the conformal function $f$ mapping $\mathcal{D}$ onto $\mathcal{G}$ with $f(0)=0$

$$
\frac{z f^{\prime}(z)}{f(z)}=\prod_{k=1}^{n}\left(1-\bar{z}_{k} z\right)^{-\beta_{k}}
$$

where the points $z_{k}$ are the preimages on $|z|=1$ of the vertices $w_{k}$ and where the following constraint conditions must be satisfied:

$$
\begin{gathered}
\beta_{k} \in\left\{-1,-\frac{1}{2}, 0, \frac{1}{2}, 1\right\}, 1 \leq k \leq n, \\
\sum_{k=1}^{n} \beta_{k}=0,
\end{gathered}
$$

and

$$
\sum_{k=1}^{n} \beta_{k} \operatorname{Arg}\left(z_{k}\right) \equiv 0 \quad(\bmod \pi) .
$$

We note that if $\mathcal{G}$ is gearlike, then it is easily seen for the mapping function $f$ we must have that $z f^{\prime}(z) / f(z)$ is either pure real or pure imaginary on the boundary of $\mathcal{D}$. If a function $f$ satisfies (i) and (ii), but not (iii), then $z f^{\prime}(z) / f(z)$ will map $\mathcal{D}$ to a domain bounded by radial segments which emanate from the origin, but which are twisted away from the coordinate axes and, hence, $f$ will map $\mathcal{D}$ to a domain which is not gearlike.

Equation (1) can be solved to explicitly represent the conformal mapping $f$ on $\mathcal{D}$ as

$$
f(z)=c z \exp \left\{\int_{0}^{z} \frac{\left[\prod_{k=1}^{n}\left(1-\bar{z}_{k} w\right)^{-\beta_{k}}-1\right]}{w} d w\right\}
$$

In the representation formula (2) the parameter $c$ and the prevertices $z_{k}, 1 \leq k \leq n$, are accessory parameters which must be determined before the conformal mapping $f$ can be computed. For each accessory parameter set satisfying (i), (ii) and (iii) a function $f$ given by (2) will map $\mathcal{D}$ onto 
a gearlike domain $f(\mathcal{D})$ whose local boundary at each vertex $w(k)=f\left(z_{k}\right)$ will be conformally homotopic to the local boundary of $\mathcal{G}$ at $w_{k}$. However, corresponding sides of $f(\mathcal{D})$ and $\mathcal{G}$ which are arcs of circles will generally not have the same modulus and corresponding sides of $f(\mathcal{D})$ and $\mathcal{G}$ which are segments of straight lines will generally not have the same argument.

Using the second formulation for uniqueness in the statement of the Riemann mapping theorem we may suppose that the prevertex $z_{n}=1$, i.e., that $f(1)=w_{n}$, and that $w_{n}$ is finite. The later assumption can always be made via a suitable reindexing of the vertices of $\mathcal{G}$. The parameter $c$ can be computed directly. If we let

$$
w^{*}=\exp \left\{\int_{0}^{1} \frac{\left[\prod_{k=1}^{n}\left(1-\bar{z}_{k} w\right)^{-\beta_{k}}-1\right]}{w} d w\right\}
$$

then

$$
c=w_{n} / w^{*} .
$$

Thus, there remain $n-1$ unknown accessory parameters to be calculated. To determine the accessory parameters we will construct a system of $n-1$ real equations, dependent on the parameters $z_{1}, \ldots, z_{n-1}$, whose solution set will be the correct accessory parameter set.

If the domain $\mathcal{G}$ is starlike, then there exists a unique function $f$ which will map $\mathcal{D}$ onto $\mathcal{G}$ as determined by the vertices $\left\{w_{k}\right\}$ and the exterior angles $\left\{\beta_{k} \pi\right\}$. However, if the domain $\mathcal{G}$ is non-starlike,there exist several functions which will map $\mathcal{D}$ onto a domain determined only by specifying the vertices $\left\{w_{k}\right\}$ and the exterior angles $\left\{\beta_{k} \pi\right\}$ - precisely because $\arg \left(w_{k}\right)$ is not uniquely determined. However, only one of these functions will be globally univalent on $\mathcal{D}$. In order to construct the univalent mapping $f$ from $\mathcal{D}$ to $\mathcal{G}$, i.e., in order to solve the accessory parameter problem for the univalent mapping, we will need to measure the total changes in argument 
over the circular sides of $\mathcal{G}$ rather than measuring the absolute arguments at the vertices of $\mathcal{G}$. We will denote the change in argument over a circular side of $\mathcal{G}$ from vertex $w_{k-1}$ to $w_{k}$ by $\Delta \arg \left(w_{k}\right)$ and we will denote the total change in argument for the mapping function $f$ over the arc (on the boundary of $\mathcal{D}$ ) from $z_{k-1}$ to $z_{k}$ by $\Delta \arg (w(k))$, where, again, $w(k)=f\left(z_{k}\right)$.

For each vertex $w_{k}, 1 \leq k \leq n-2$, there are four (mutually exclusive) possibilities that can arise:

(a) the vertex $w_{k}$ lies at infinity;

(b) the vertex $w_{k}$ is finite and lies on a radial side of $\mathcal{G}$ which comes in from infinity (as the boundary of $\mathcal{G}$ is traversed positively), but not at the end of the radial side, i.e., the interior angle at $w_{k}$ is $\pi$;

(c) the vertex $w_{k}$ is finite and lies at the end of a radial side of $\mathcal{G}$, which comes in from infinity;

(d) the vertex $w_{k}$ is otherwise finite.

If (a) holds, then we will not impose any condition on $w(k)$; however, the geometry of $\mathcal{G}$ will force the next vertex to be finite.

If (b) holds, then we will impose a modulus condition on $w(k)$ and require that

$$
|w(k)|-\left|w_{k}\right|=0 .
$$

If (c) holds, then we will impose two conditions on $w(k)$. We will require that the modulus condition (3a) holds and we will require that

$$
\operatorname{Arg}(w(k))-\operatorname{Arg}\left(w_{k}\right)=0
$$

In the last case, then either $\left|w_{k}\right|=\left|w_{k-1}\right|$ or else $\operatorname{Arg}\left(w_{k}\right)=\operatorname{Arg}\left(w_{k-1}\right)$. If $\left|w_{k}\right|=\left|w_{k-1}\right|$, then we will require

$$
\Delta \arg (w(k))-\Delta \arg \left(w_{k}\right)=0
$$


otherwise we will require (3a) to hold.

The above construction will generate $n-2$ equations if the vertex $w_{n-2}$ does not lie at infinity. If, however, $w_{n-2}$ does lie at infinity, then one additional equation will need to be generated. The vertex $w_{n-1}$ must be finite and satisfy condition (c). Thus, we can add one equation to the system by adding the modulus equation (3a) with $k=n-1$.

The system of equations generated at this point may not be sufficient to characterize the accessory parameter set for the correct mapping function, especially in the case that $\mathcal{G}$ is unbounded and non-starlike. We may need to replace one of the equations in the system with a secondary equation to control the total argument change for the mapping function or to control the location of the tip of a slit. There are three cases which need to be considered.

Case 1. The vertex $w_{n-1}$ or $w_{n}$ lies at the end of a radial side of $\mathcal{G}$ going out to infinity and the interior angle at that end is $\pi / 2$. If $w_{n-1}$ lies at the end of the radial side, then the last equation in the system of type (3b) must be replaced by an argument condition (3c) with $k=n-1$. Alternately, if $w_{n}$ lies at the end of the radial side, then either $w_{n-1}$ is the tip of a circular slit or the boundary of $\mathcal{G}$ has a right angle corner at $w_{n-1}$. If $w_{n-1}$ is the tip of a circular slit, then the last equation in the system of type (3a) must be replaced by an argument condition (3c) with $k=n$; otherwise, the last equation in the system of type (3b) must be replaced by an argument condition (3c) with $k=n$.

Case 2. Case 1 does not apply and condition (c) holds for some vertex $w_{k_{0}}, 1 \leq k_{0} \leq n-1$, such that $w_{k_{0}}$ lies on the component of the boundary of $\mathcal{G}$ which contains $w_{n}$ and the boundary of $\mathcal{G}$ has a right angle corner at $w_{k}$. If $w_{n-1}$ is the tip of a radial slit, then the argument equation (3b) generated by condition (c) at $k=k_{0}$ must be replaced by a modulus condition (3a) with $k=n-1$. Alternatively, if $k_{0}=n-1$, then the modulus equation (3a) generated by condition (c) at $k=k_{0}$ must be replaced by an argument 
condition (3c) with $k=n$. Alternately, if $w_{n-1}$ lies at the tip of a circular slit, then the last equation in the system of type (3a) must be replaced by an argument condition (3c) with $k=n-1$. Otherwise, if the segment on the boundary of $\mathcal{G}$ between $w_{n-2}$ and $w_{n-1}$ is a circular arc, then the argument equation (3b) generated by condition (c) at $k=k_{0}$ must be replaced by an argument condition (3c) with $k=n-1$.

Case 3. The domain $\mathcal{G}$ is bounded and the vertex $w_{n-1}$ lies at the tip of a slit. The above construction does not completely characterize the geometry of $\mathcal{G}$, i.e., the generated mapping function $f$ may not satisfy $f\left(z_{n-1}\right)=w_{n-1}$. The last equation of the system, that is, the equation representing the vertex $w_{n-2}$, must be replaced by a condition determined by the geometry at the vertex $w_{n-1}$. If $w_{n-1}$ lies at the tip of a radial slit, then we will require that (3a) holds for $k=n-1$, otherwise we will require that (3c) holds for $k=n-1$.

To determine the full set of $n-1$ accessory parameters we need to add one additional equation to the above system of equations. We have not yet imposed the constraint condition (iii) in the representation formula for gearlike mappings. While we could solve (iii) for one of the prevertices (in terms of the others) and thus reduce the number of unknown accessory parameters from $n-1$ to $n-2$, our computational experience has been that we have been able to treat a variety of problems (i.e., solve the accessory parameter problem) using a full set of $n-1$ equations, with its extra degree of freedom, which we could not successfully treat using a reduced set of $n-2$ equations. We will, therefore, impose (iii) as the $n-1^{\text {st }}$ system equation.

Let us note that if the interior angle at $w_{n-1}$ is $\pi$, then the generated mapping $f$ may not satisfy $f\left(z_{n-1}\right)=w_{n-1}$. No auxiliary condition can be added to the above construction without over-determining the problem. On the other hand, the point $w_{n-1}$ can be removed from the set of vertices of $\mathcal{G}$ without changing the geometry of $\mathcal{G}$. We will require, in the presentation of 
the vertices of $\mathcal{G}$, that $\beta_{n-1}$ be not 0 .

\section{COMPUTATION}

In the actual practice of solving the nonlinear system of equations (3) and computing the conformal mapping $f$, we have generally employed the techniques described by Trefethen. (See [Tr],[ pp. 86-90].) The given form (3) requires solving for the unknown complex prevertices $z_{k}$ on the unit circle $|z|=1$. (As with the vertices, we will also denote $z_{n}$ as $z_{0}$.) It is numerically more convenient to transform the points $z_{k}$ to their arguments $\theta_{k}$ by

$$
z_{k}=e^{i \theta_{k}}, 0 \leq \theta_{k} \leq 2 \pi
$$

The arguments $\theta_{k}$ are, however, severely constrained by a set of linear inequalities because the points $z_{k}$ are ordered around the unit circle. The ordering constraints on (3) can be removed by transforming the arguments $\theta_{k}$ to a set of unconstrained variables $y_{k}$ via the equations

$$
y_{k}=\log \frac{\theta_{k}-\theta_{k-1}}{\theta_{k+1}-\theta_{k}}, 1 \leq k \leq n-1,
$$

where we take $\theta_{0}$ to be $\operatorname{Arg}\left(z_{0}\right)=0$. Because the arguments $\theta_{k}$ in (4) are coupled, the transformation can be inverted to recover the arguments $\theta_{k}$ from the images $y_{k}$.

At each step in the iteration we will calculate from the unconstrained variables $y_{k}, 1 \leq k \leq n-1$, a set of arguments $\theta_{k}$ and then a set of prevertices $z_{k}$. (The prevertex $z_{n}$ is fixed at 1.) Finally, we will calculate the values of the $n-1$ nonlinear equations (3) for the current set of prevertices.

In the computation of the values $f\left(z_{k}\right)$, we generally choose a path for the integration in (2) which is the straight line segment $\left[0, z_{k}\right]$ in $\mathcal{D}$. The integrand in (2) will have a singularity at both 0 and at $z_{k}$. However, the singularity at 0 is removable and can be controlled directly. The singularity at $z_{k}$ is of the form $\left(1-\bar{z}_{k} z\right)^{-\beta_{k}}$ where $\beta_{k}$ can take on only one of five possible values. Normally, such a singularity in an integral could be easily, 
and highly accurately, treated by Gauss-Jacobi quadrature. However, there may be, and typically there are, other prevertices $z_{j}$ clustered near $z_{k}$, which could affect the accuracy of the quadrature result. Trefethen described a type of compound Gauss- Jacobi quadrature (see [Tr], [p. 87]) which divides the path of integration into subpaths, where the length of each subpath is dependent on how closely other prevertices $z_{j}$ are clustered to $z_{k}$. This type of compound Gauss-Jacobi quadrature has produced both highly accurate and efficient quadrature results.

We have used the library subroutine GAUSSQ by G. H. Golub and J. H. Welsch [Go \& We] to calculate the nodes and weights for the GaussJacobi quadrature. We have usually set the number of nodes, NPTSQ, to be computed by GAUSSQ at 8. Since the compound Gauss- Jacobi quadrature always divides the path of integrations into two halves and one of the halves may be further subdivided, dependent on the distribution of the current set of prevertices $z_{k}$, we achieve, in practice, at least 16 nodes for integration on each path.

To solve the system of unconstrained nonlinear equations we have used the library subroutine NS01A by M. J. D. Powell [Po], which employs a hybrid between the method of steepest descent (initially) and Newton's method (terminally).

While the solution driver which calls NS01A can pass specialized initial sets of prevertices, should such information be available, the general initialization does not take into account any information about the geometry of the particular gearlike conformal mapping problem. In general, the initialization allocates a set of initial prevertices uniformly distributed around the unit circle.

Two of the control parameters required by NSO1A are: DSTEP, the step size used by NS01A to calculate the Jacobian of (3) by finite difference methods - generally fixed at $10^{-8}$ and TOL, the convergence criterion for returning from NS01A to the calling program after having successfully solved 
(3). The value of TOL is closely related to the number of nodes NPTSQ selected for the compound Gauss-Jacobi quadrature. For a problem with vertices close to unit norm TOL is typically set at $10^{-(\mathrm{NPTSQ}+1)}$.

Once the accessory parameters have been determined, values of the mapping function $f$ can be computed for given initial points $z$ in $\mathcal{D}$ or on the boundary of $\mathcal{D}$. In either case, the values are computed by using compound Gauss-Jacobi quadrature. While the value of $f$ at a point $z$ can always be computed using (2) directly, if $z$ is near a point $z^{*}, z^{*} \neq 0$, where the value of $f$ is known, then the value $f(z)$ can be alternately computed as

$$
f(z)=\frac{f\left(z^{*}\right)}{z^{*}} z \exp \left\{\int_{z^{*}}^{z} \frac{\left[\prod_{k=1}^{n}\left(1-\bar{z}_{k} w\right)^{-\beta_{k}}-1\right]}{w} d w\right\} .
$$

The point $z^{*}$ can be one of the prevertices corresponding to a known finite vertex of $\mathcal{G}$ or the point $z^{*}$ can be point where the value of $f$ has been previously computed.

Values for the inverse mapping function from the gearlike domain $\mathcal{G}$ to the unit disk $\mathcal{D}$ can also be generated. Let $w$ be in $\mathcal{G}$ or on the boundary of $\mathcal{G}$. If, for $z=f^{-1}(w)$, a nearby initial estimate $z^{*}$ can be given, then Newton's method can be employed to solve for $z$. (The value of the derivative of $f$ can be calculated from equation (1).) On the other hand, when no initial estimate $z^{*}$ is known, we can rewrite equation (1) as

$$
\frac{d w}{d z}=\frac{w}{z} \prod_{k=1}^{n}\left(1-\bar{z}_{k} z\right)^{-\beta_{k}}
$$

where $w=f(z)$. The univalence of the mapping function implies that equation (5) can inverted to obtain

$$
\frac{d z}{d w}=\frac{z}{w} \prod_{k=1}^{n}\left(1-\bar{z}_{k} z\right) .{ }^{+\beta_{k}}
$$


Equation (6) can be viewed as an ordinary differential equation which lifts a straight line segment $[\tilde{w}, w]$ in $\mathcal{G}$ to a solution curve in $\mathcal{D}$ from a point $\tilde{z}=f^{-1}(\tilde{w})$ to the point $z$. The point $\tilde{w}$ can be any point in $\mathcal{G}$ provided that the straight line segment $[\tilde{w}, w]$ lies entirely in $\mathcal{G}$. To solve the ordinary differential equation we have used the library subroutine ODE by Shampine and Gordon [Sh \& Go]. The code in ODE treats real differential equations, which requires that we transform (6) to a coupled pair of real differential equations.

We have, following Trefethen, combined the above two approaches, unless an initial estimate is known for which Newton's method can be applied directly. We will first solve (6), starting typically at $\tilde{w}=0$, to obtain a low order approximation $z^{*}$ to $z$. We will then follow up using Newton's method to move from $z^{*}$ to a final approximation of $z$ with high order accuracy.

It is important that not only are we able to construct the approximate numerical mapping by solving the accessory parameter problem, but that we are able to estimate the accuracy of the approximation we have generated. After the nonlinear solver NS01A returns a solution for the accessory parameter problem we check the accuracy of the computed solution by checking at each finite node $w_{k}$ the difference $\left|w_{k}-w(k)\right|$. At each infinite node $w_{k}$ we check the difference ||$w_{k-1}-w_{k+1}|-| w(k-1)-w(k+1) \|$. An error routine returns the maximum of the above tested differences as an error estimate for the accuracy of the generated mapping function.

\section{EXAMPLES}

In Figure 3 we show conformal mappings generated at several of the iterative steps which arise in constructing the conformal mapping in Figure 2. We start the process with no assumption about the geometry of the prevertices for the image domain $\mathcal{G}$ in Figure 2, i.e., we begin with an initial uniform

distribution about the unit circle for the prevertices. Also, we have set the number of nodes for the compound Gauss-Jacobi quadrature at NPTSQ $=8$ 
and have set the level of desired accuracy at TOL $=10^{-8}$. The plots in Figure 3 show several contours (images of circles centered at the origin) and the images of the six radial segments which join the origin to the prevertices. As Trefethen noted, the conformality of the mappings at the origin can be used to interpolate on the plots in Figure 3 the arguments of the prevertices via the arguments of the images of the radial segments at they approach the origin. The example in Figure 3 is typical in that it shows some of the prevertices to be clustering as the iterations move towards the mapping solution at stage I20.

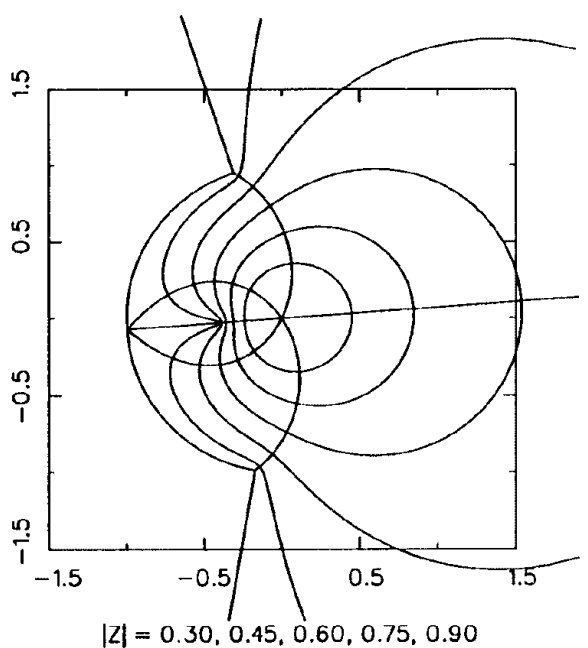

I5

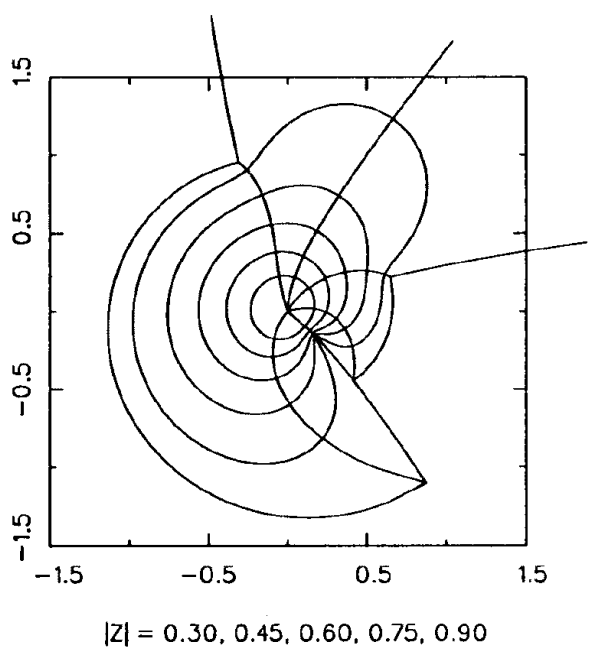

I7

Figure 3 


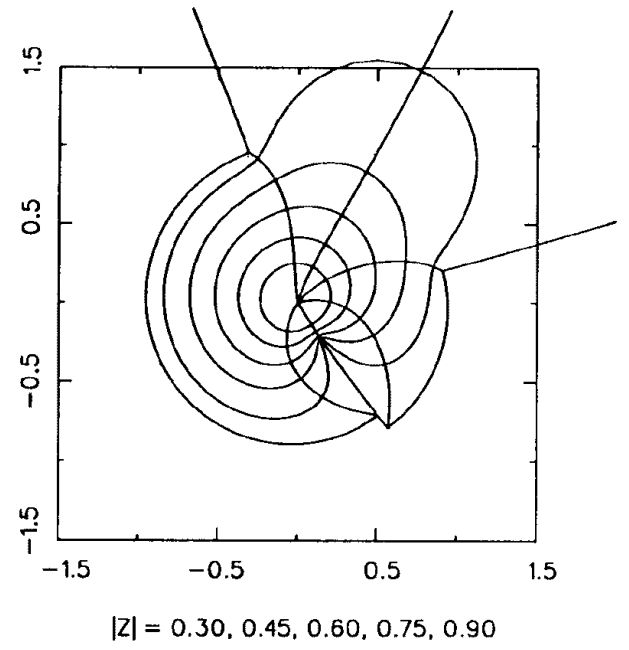

I8

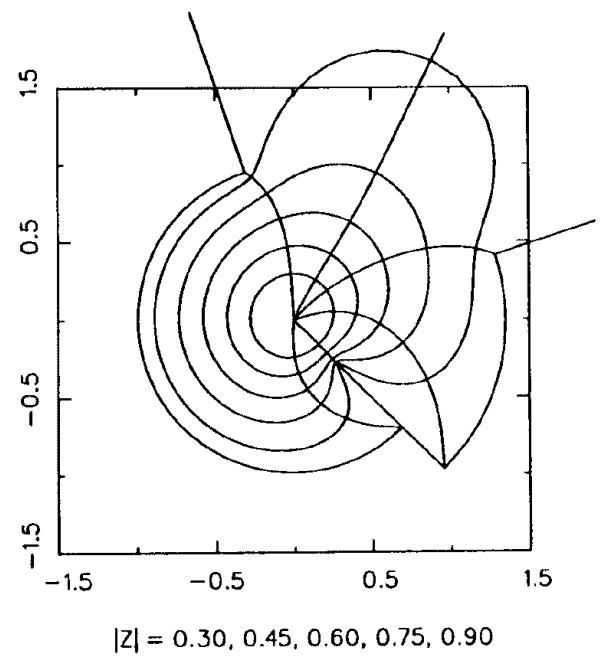

I11

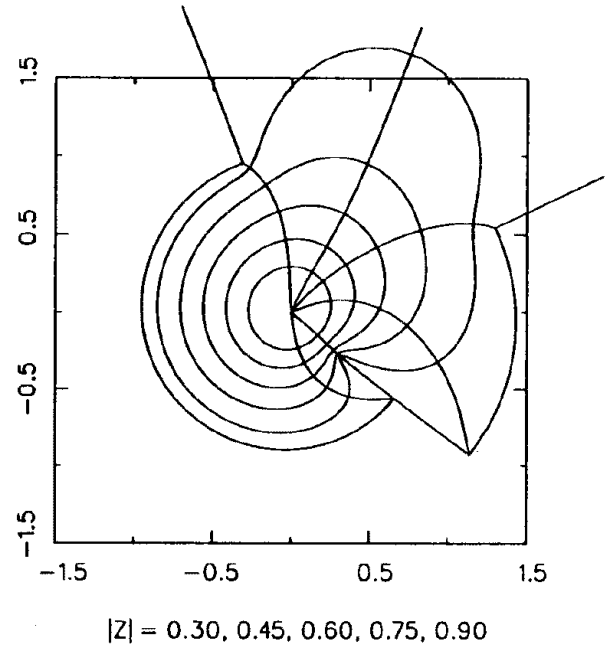

I9

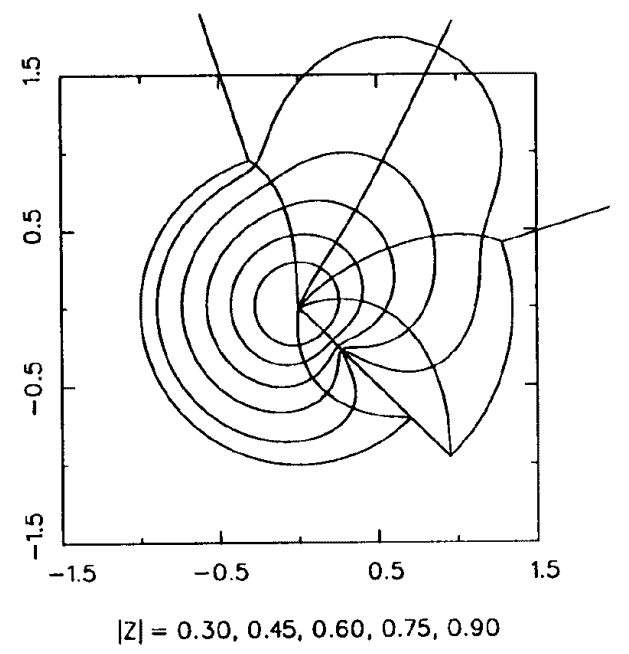

I20

Figure 3 (cont.)

In Figures 4 and 5 we show examples which illustrate the dispersion of the contours for several sets of bounded and unbounded gearlike domains. In each case the contours are the images of the circles $|z|=r, 0<r<1$.

In Figures 6 and 7 we show examples which illustrate the dispersion of 
the level sets for the inverse mappings for the gearlike domains depicted in Figures 4 and 5. In each case the level sets are the images under $f^{-1}$ of $|w|=R, R>0$. The ticks shown on the boundary of $\mathcal{D}$ mark the location of the prevertices $z_{k}$.

Finally, let us note one difficulty which we have encountered. The only requirement for applying the above algorithm to a gearlike domain mapping problem is that one of the finite vertices must be distinguished as the last vertex, $w_{n}$, (and, or course, then $w_{n-1}$ must not be removable). There may be for a given gearlike domain several vertices which could be so distinguished. Our experience, however, in implementing the algorithm, i.e., in solving the nonlinear system of parameter equations via NS01A, has been that frequently only one of the several choices (for the distinguished vertex) has led to a tractable system of equations. 


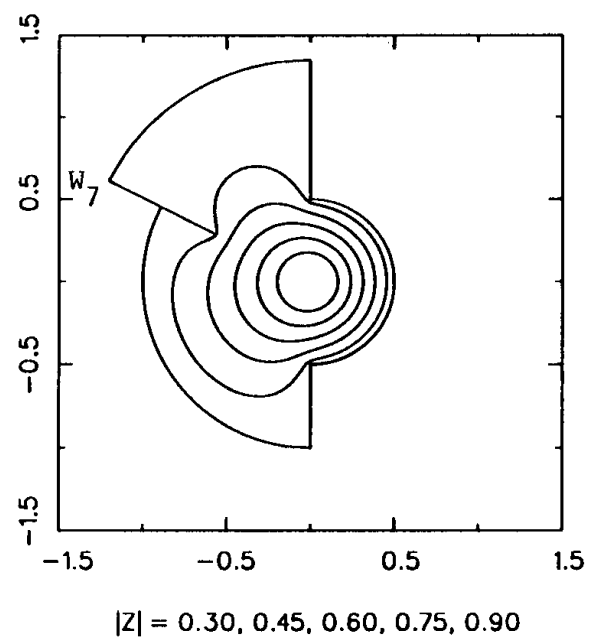

(a)

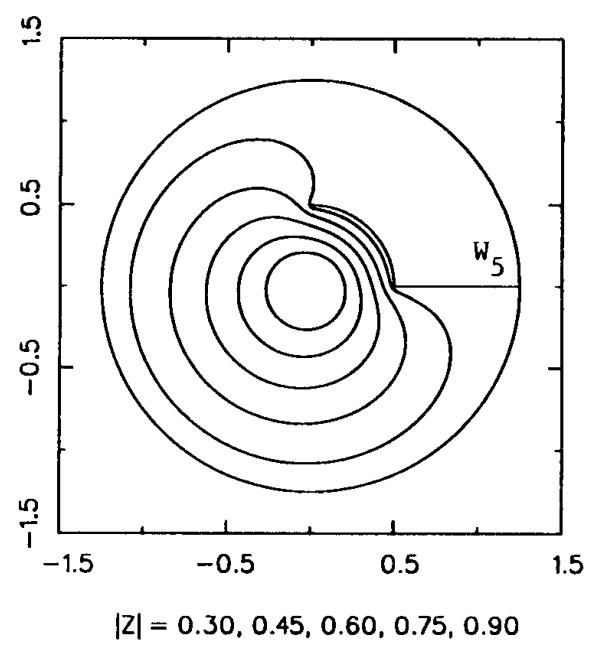

(c)

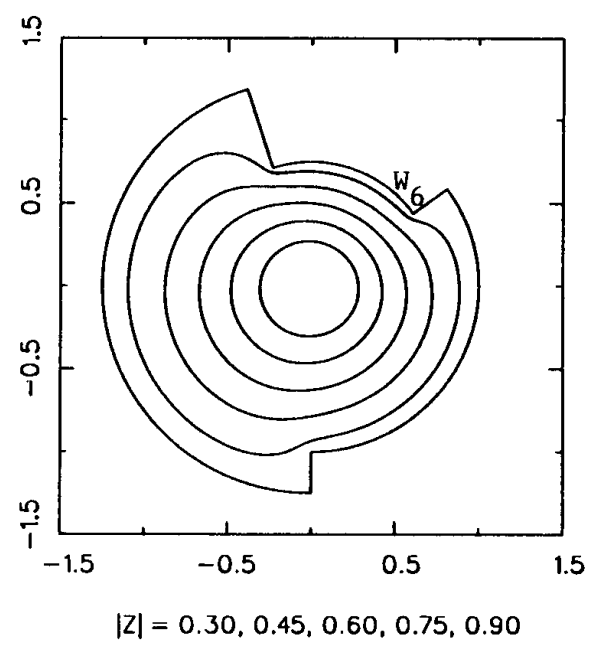

(b)

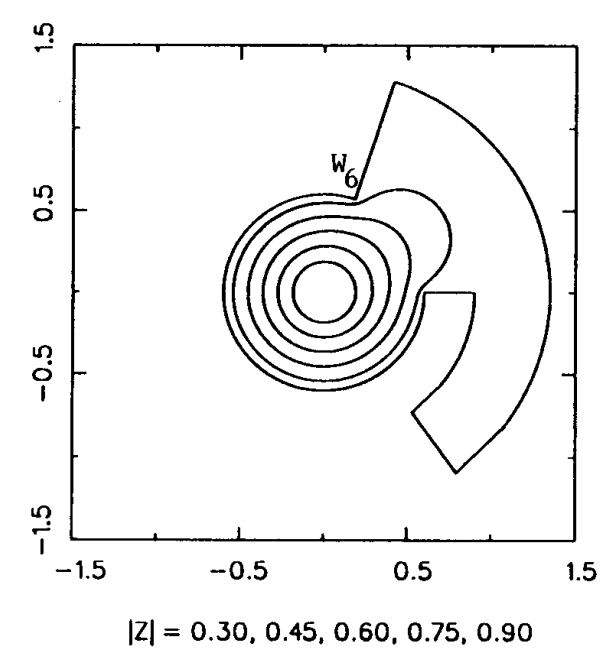

(d)

Figure 4 


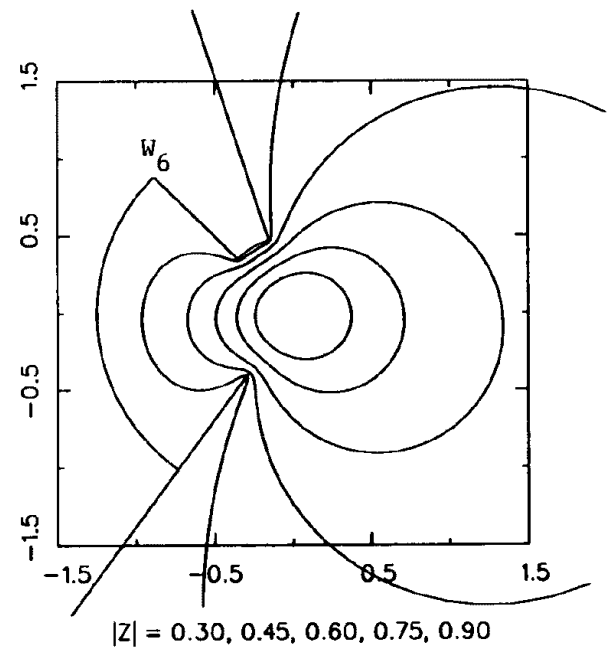

(a)

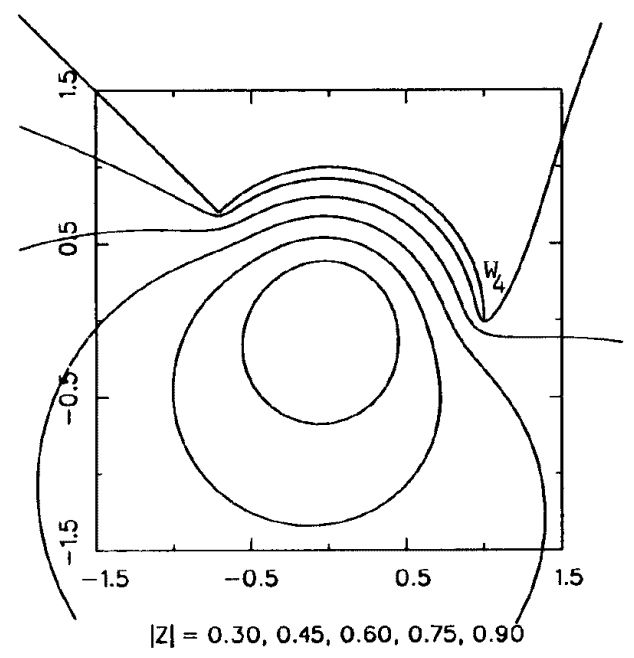

(c)

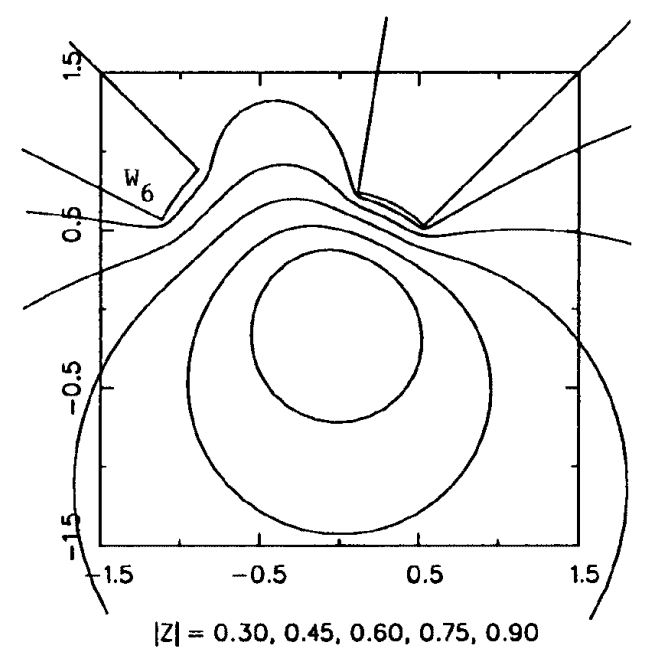

(b)

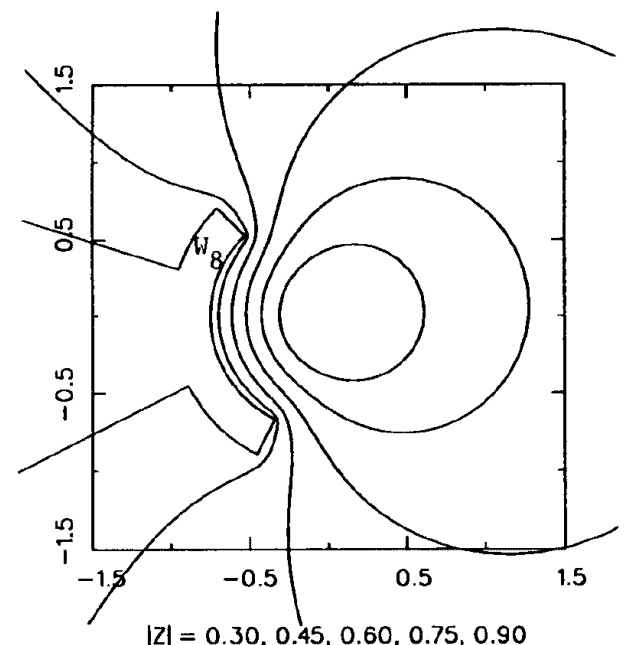

(d)

Figure 5 


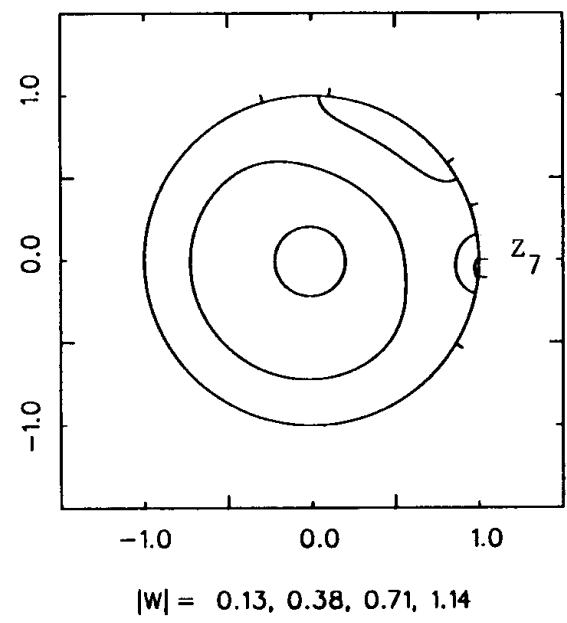

(a)

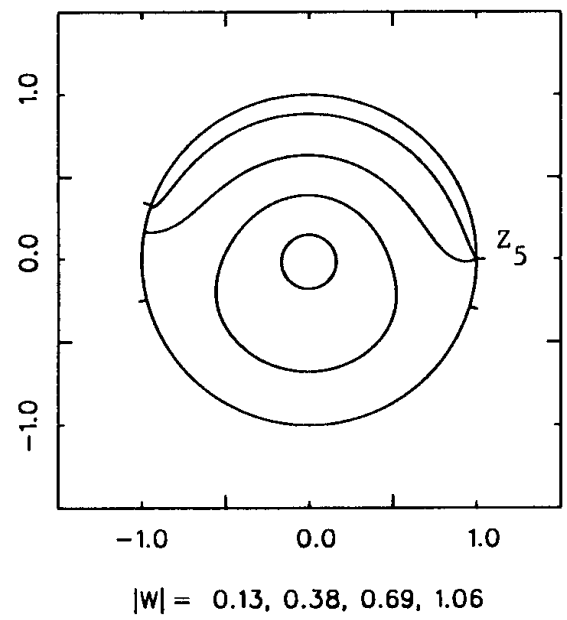

(c)

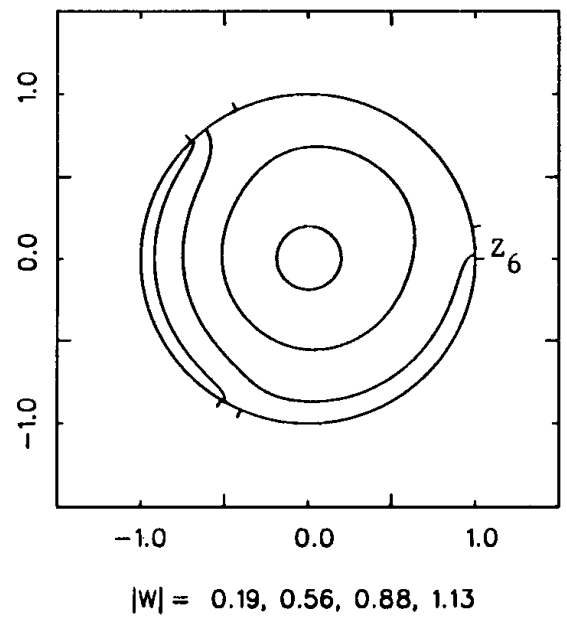

(b)

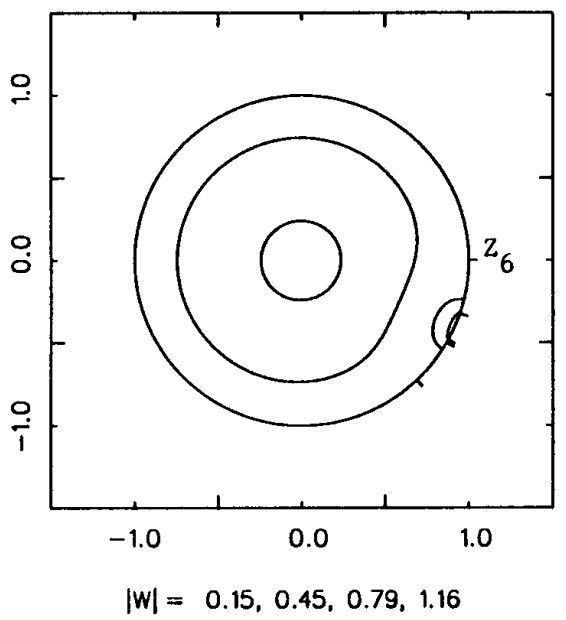

(d)

Figure 6 


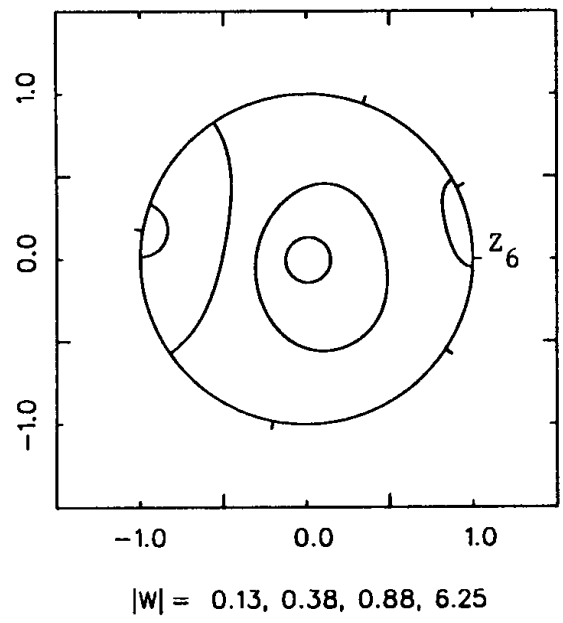

(a)

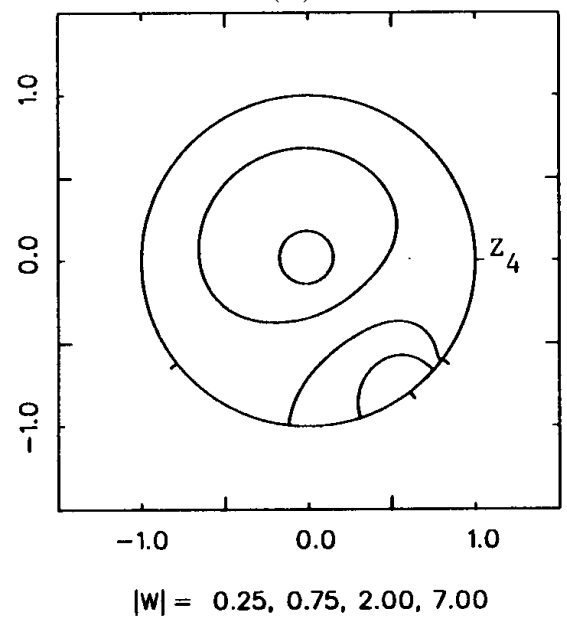

(c)

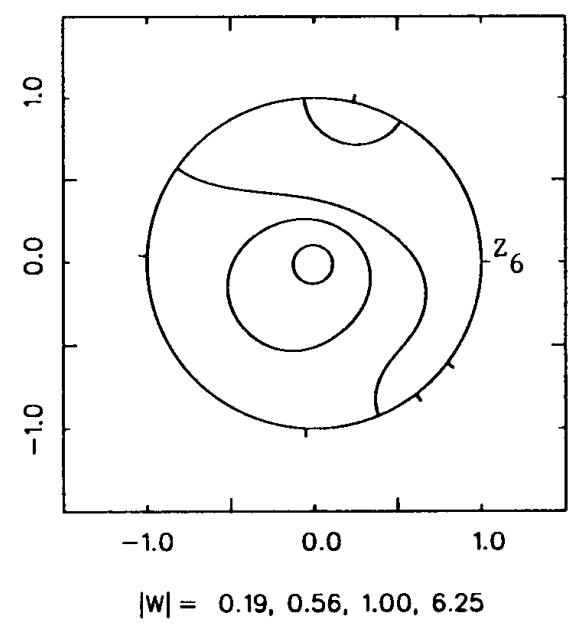

(b)

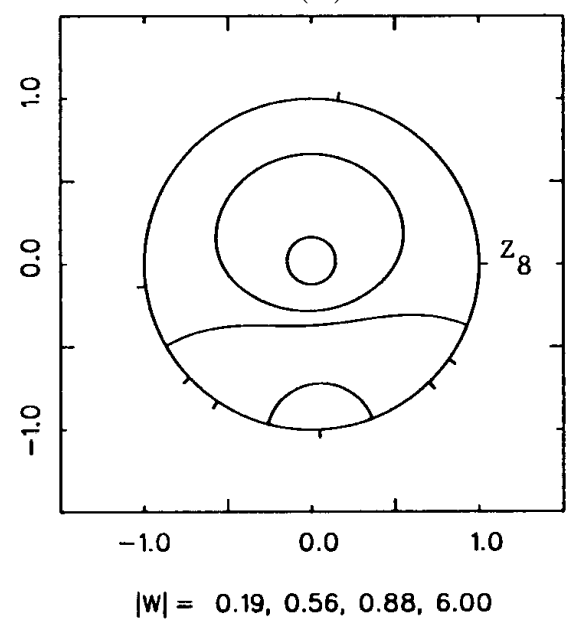

(d)

Figure 7 


\section{APPLICATION}

Let $S t$ be the class of analytic univalent functions $f$ on $\mathcal{D}$, normalized by $f(0)=0$ and $f^{\prime}(0)=1$, which map $\mathcal{D}$ to an image domain which is starlike with respect to the origin. For $M \geq 1$ let $\mathcal{D}(M)=\{z|| z \mid<M\}$ and let $S t(M)$ be the subclass of $S t$ of functions which satisfy the property that $f(\mathcal{D}) \subset \mathcal{D}(M)$. Let $f \in S t(M)$ and have a power series representation

$$
f(z)=z+\sum_{n=2}^{\infty} a_{n} z^{n} .
$$

R. W. Barnard and J. L. Lewis [Ba], [Ba \& Le] considered the problem of finding

$$
\max _{f \in \operatorname{St}(M)} \mathrm{Rea}_{3} .
$$

They showed that if $M \geq 5$ and if $f$ is extremal for the coefficient problem (7), then $f$ is the Pick function in $S t(M)$, i.e., $f$ maps $\mathcal{D}$ onto the disk $\mathcal{D}(M)$ minus a radial slit on the negative real axis. (See Figure 8a.) If $1<M \leq e$, they showed that if $f$ is extremal for (7), then $f$ is the SRT (square root transform) Pick function in $S t(M)$, i.e., $f$ maps $\mathcal{D}$ onto the disk $\mathcal{D}(M)$ minus a pair of symmetric radial slits on the imaginary axis. (See Figure 8b.)

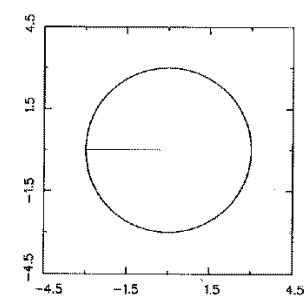

a

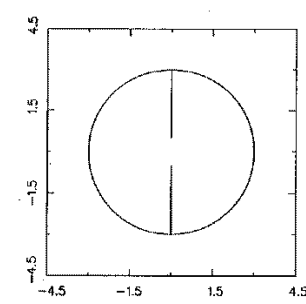

$\mathrm{b}$

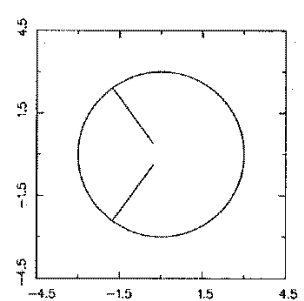

C

Figure 8

While they were not able to solve the extremal problem for $e<M<5$, they were able to show that the extremal function must map $\mathcal{D}$ onto $\mathcal{D}(M)$ 
minus a pair of real-axis symmetric radial slits. (See Figure 8c.) They noted that if $M=3$, then the third coefficient for the Pick function in $\operatorname{St}(M)$ and the third coefficient for the SRT Pick function in $S t(M)$ are the same. Lewis posed the coefficient problem (7) for $e<M<5$ as Problem 6.65 in $[\mathrm{Ca}, \mathrm{Cl} \& \mathrm{Ha}]$ and Barnard conjectured [Ba] for $M \geq 3$ that (7) was maximized by the Pick function in $S t(M)$ and for $1<M \leq 3$ that (7) was maximized by the SRT Pick function in $S t(M)$.

To support that conjecture the problem was posed to show numerically for $e<M<5, M$ fixed, and for the real-axis symmetric double radial slit mappings in $S t(M)$ that $\operatorname{Rea}_{3}$ was concave as a function of the argument $\alpha$ of the omitted slit, $\pi / 2 \leq \alpha \leq \pi$. Each such function $f$ is a bounded gearlike mapping and can be represented by (from the geometry of the image domain)

$$
\frac{z f^{\prime}(z)}{f(z)}=\sqrt{\frac{p_{2}^{2}(z)}{p_{1}(z) p_{3}(z)}}=Q(z)
$$

where $p_{j}(z)=1-2 x_{j} z+z^{2}, x_{j}=\operatorname{Re} z_{j}=\cos \theta_{j}, z_{j}=e^{i \theta} j$ and $0 \leq \theta_{1} \leq$ $\theta_{2} \leq \theta_{3} \leq \pi$. If we let

$$
Q(z)=1+\sum_{k=1}^{\infty} q_{k} z^{k}
$$

then

$$
a_{3}=\frac{q_{1}^{2}+q_{2}}{2}
$$

where

$$
\begin{aligned}
& q_{1}=x_{1}+x_{3}-2 x_{2} \\
& q_{2}=\frac{3\left(x_{1}+x_{3}\right)^{2}-4\left(x_{1} x_{2}+x_{2} x_{3}+x_{3} x_{1}\right)}{2} .
\end{aligned}
$$

We specifically considered the case $M=3$. As we constructed the values of $R e a_{3}$, for incremental values of argument $\alpha$ of the omitted slit, we found (using a value for $T O L=10^{-8}$ ), however, (see Table 1) that the conjecture was not correct. Indeed, Table 1 suggests for $M=3$ that $R e a_{3}$ is unimodal 
as a function of $\alpha$ over the interval $[\pi / 2, \pi]$ and that the maximum value occurs for some $\alpha$ in $[0.80 \pi, 0.90 \pi]$.

We considered various values for $M$ and found evidence to suggest that the argument $\alpha$ of the omitted slit for the extremal function for (7) appears to vary monotonically with $M$ from $\pi / 2$ to $\pi$ as $M$ varies from $e$ to 5 .

Table 1

$\begin{array}{cc}\alpha / \pi & \text { Rea }_{3} \\ & \\ 0.500 & 0.88888889 \\ 0.550 & 0.89011287 \\ 0.600 & 0.89358764 \\ 0.650 & 0.89873157 \\ 0.700 & 0.90461073 \\ 0.750 & 0.91000424 \\ 0.800 & 0.91353195 \\ 0.850 & 0.91388099 \\ 0.900 & 0.91009490 \\ 0.950 & 0.90174616 \\ 1.000 & 0.88888889\end{array}$

Barnard remarked, after seeing the numerical results in Table 1, that it should be possible to show analytically that the conjecture was false. Indeed, a long, extensive calculus argument can be given which shows that the conjecture fails for $2.83912<M \leq 3$. Specifically, the argument shows for $2.83912<M$ that $R_{e} a_{3}$ takes on a local minimum value at the SRT Pick function over the set of real-axis symmetric double radial slit mappings in $\operatorname{St}(M)$.

We would like to thank Professor R. W. Barnard his helpful comments and suggestions during the preparation of this paper. 


\section{References}

[Ba] R. W. Barnard, "A variational technique for bounded starlike functions," Can. J. Math. 2 (75) 337-347.

[Ba \& Le] R. W. Barnard and J. L. Lewis, "Coefficient bounds for some classes of starlike functions," Pac. J. Math. 75 (1975), 325-331.

[Ba \& Pe] R. W. Barnard and K. Pearce, "Rounding corners of gearlike domains and the omitted area problem," J. Comp. Appl. Math. 14 (1986), 217-226.

[Bj \& Gr] P. Bjørstad and E. Grosse, "Conformal Mapping of Circular Arc Polygons," SIAM J. Sci. Stat. Comp., to appear.

[Ca, Cl \& Ha] D. M. Campbell, J. G. Clunie and W. K. Hayman, "Research problems in complex analysis," Aspects of contemporary complex analysis, (Proc. NATO Adv. Study Inst., Univ. Durham, 1979), 527-572, Academic Press, London, 1980.

[Ga] D. Gaier, Konstruktive Methoden der konformen Abbildung, Springer Verlag, Berlin, 1964.

[Go \& We] G. H. Golub and J. H. Welsch, "Calculation of Gaussian quadrature rules," Math. Comp. 23 (1969), 221-230.

[Go] A. W. Goodman, "Conformal mapping onto certain curvilinear polygons," Univ. Nac. Tucuman A 13 (1960) 20-26.

[He] P. Henrici, Applied and computational complex analysis, Vol. III, John Wiley \& Sons, New York, 1986. 
[Ja \& Ma] K. P. Jackson and J. C. Mason, "The Approximation by Complex Functions of Stresses in Cracked Domains," Algorithms for Approximation, Oxford University Press, 1987.

[Ne] Z. Nehari, Conformal Mapping, McGraw-Hill Book Co., Inc., New York, 1952.

[Po] M. J. D. Powell, Technical Report AERE-R.5947, Harwell, England, 1968.

[Sh \& Go] L. Shampine and M. Gordon, Computer solution of ordinary differential equations: The initial value problem, Freeman, San Francisco, 1975.

[Tr] L. N. Trefethen, "Numerical computation of the SchwarzChristoffel transformation," SIAM J. Sci. Stat. Comp. 1 (1980), 82-102.

[Tr, 83] L. N. Trefethen, "Radial and Gearlike Domains," unpublished memo, 1983. 